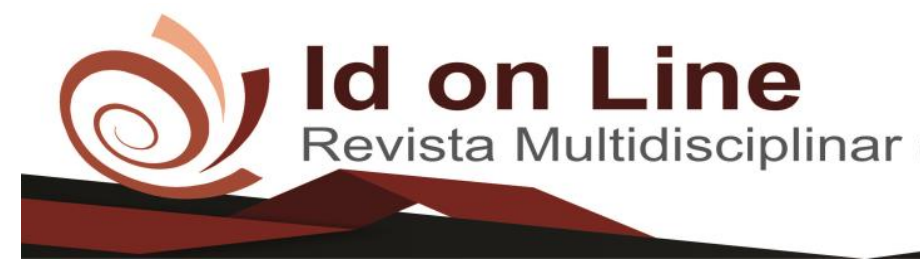

DOI: 10.14295/idonline.v15i54.2960

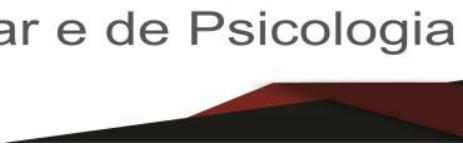

Artigo de Revisão

\title{
Aplicação das Práticas Integrativas e Complementares na Atenção Primária à Saúde
}

\author{
Mariana dos Santos Araujo Vidal, Milena Nunes Alves de Sousa, Miguel Aguila Toledo
}

\begin{abstract}
Objetivo: Avaliar quais os problemas de saúde abordados pelas Práticas Integrativas e Complementares na Atenção Primária à Saúde. Métodos: Consiste em uma revisão integrativa de literatura, a qual possibilita a construção de um panorama da produção científica disponível acerca do tema, com análise dos artigos publicados nos últimos cinco anos em idioma português, nas bases de dados da Biblioteca Virtual em Saúde e da Biblioteca Nacional de Medicina dos Estados Unidos, com seleção final de 14 artigos para avaliação integral. Resultados: Foram observadas cinco categorias de agravos à saúde nas quais houve aplicação das Práticas Integrativas e Complementares, sendo elas a saúde mental, manejo da dor, doenças crônicas, doenças neurológicas e menopausa. Conclusão: Apesar dessas práticas apresentarem seu uso disseminado em algumas patologias, ainda há uma carência de estudos com foco nas suas aplicações e respectivos resultados na situação de saúde dos indivíduos.
\end{abstract}

Palavras-Chave: Terapias Complementares, Atenção Primária à Saúde, Terapêutica.

\section{Application of Integrative and Complementary Practices in Primary Health Care}

Objective: Assess which health problems have application Integrative and Complementary Practices in Primary Health Care. Methods: It consists of an integrative literature review, which allows the construction of a panorama of the scientific production available on the subject, with analysis of the articles published in the last five years in Portuguese, in the databases of the Virtual Health Library and the National Library of Medicine of the United States, with a final selection of 14 articles for full evaluation. Results: Five categories of health problems were observed in which the Integrative and Complementary Practices were applied, namely mental health, pain management, chronic diseases, neurological diseases and menopause. Conclusion: Although these practices have their widespread use in some pathologies, there is still a lack of studies focusing on their applications and respective results in the health situation of individuals.

Keywords: Complementary therapies, Primary Health Care, Therapeutics.

\footnotetext{
${ }^{1}$ Médica residente em Medicina da Familía e Comunidade . UNIFIP. marianaaraujo96@ hotmail.com;

${ }^{2}$ Doutorado e PósDoutorado em Promoção da Saúde. Centro Universitário de Patos e Faculdade São Francisco da Paraíba. milenanunes@fiponline.edu.br;

${ }^{3}$ Mestrado em Infectologia. Centro Universitário de Patos. migueltoledo@fiponline.edu.br.
} 


\section{Introdução}

As Práticas Integrativas e Complementares em Saúde (PICS), ou, como também são chamadas, Medicina Tradicional e Complementar (MTC), constituem um grupo de práticas com uma abordagem diferenciada em relação à medicina convencional ocidental, que segue o modelo biomédico (TESSER, 2009). Essas práticas consistem em uma diversidade de sistemas médicos que buscam a promoção da saúde e a recuperação terapêutica através de mecanismos naturais e eficazes, que contemplem de forma integral o indivíduo através de uma visão ampliada sobre seu processo saúde doença (BARBOSA, 2020).

No Brasil, essas práticas são regulamentadas e estimuladas no âmbito da saúde pública através da Política Nacional de Práticas Integrativas Complementares (PNPIC) do Sistema Único de Saúde (SUS), que teve sua primeira publicação na íntegra em 2006, em resposta a um cenário mundial no qual vários documentos sobre o tema já vinham sendo publicados e a implantação dessas práticas já era estimulada pela Organização Mundial de Saúde (OMS) desde a década de 70, e também a um cenário nacional onde essa práticas já vinham sendo desenvolvidas, principalmente após a criação do SUS na década de 80 (MINISTÉRIO DA SAÚDE 2015). Segundo a última atualização, quanto às práticas contempladas na PNPIC temse: medicina tradicional chinesa/acupuntura; fitoterapia; crenoterapia; medicina antropofásica; aromaterapia; imposição de mãos; hipnoterapia; entre outras (MINISTÉRIO DA SAÚDE, 2018).

Essa valorização das PICS no cenário científico ocorreu principalmente a partir da transição epidemiológica, com o envelhecimento da população e consequente destaque das doenças crônicas (GALVANESE; BARROS; OLIVEIRA, 2017), as quais envolvem um processo de adoecimento singular para cada paciente, com potencial para alterar seu modelo de vida e percepção de saúde. Além disso, a globalização e o aumento de transtornos mentais também estimularam a busca pelas PICS como opção terapêutica e promotora de saúde (SOUSA; HORTALE; BODSTEIN, 2018). A mudança que estas novas formas de adoecimento da sociedade atual encontraram barreiras no modelo biomédico na medida em que necessitam de uma visão mais integral da saúde, com foco não só na doença e sim no indivíduo, e com a perspectiva não de cura, mas de melhora da qualidade de vida e alívio dos sintomas.

Foram características intrínsecas às PICS e comuns às suas diferentes práticas, como a integralidade, flexibilidade, individualização, e potencial de complexidade que permitiram seu avanço e disseminação nos serviços de saúde (SOUSA; HORTALE; BODSTEIN, 2018). 
Foram essas características também que fizeram da atenção primária à saúde (APS) seu principal espaço de prática na saúde pública brasileira (BARBOSA, 2020). A integralidade do cuidado, acolhimento e a longitudinalidade da atenção tornaram a APS o local adequado para o desenvolvimento das PICS. Segundo estudo realizado por Barbosa (2020), que analisou a oferta de PICs no Brasil a partir de dados do Programa Nacional de Melhoria do Acesso e da Qualidade da Atenção Básica (PMAQ) e do Inquérito Nacional de Práticas Integrativas e Complementares em Saúde no SUS, com base no Inquérito, dos municípios com oferta de PICS, na maioria $(70,8 \%)$ deles a oferta estava presente na ESF.

Apesar de já estar bem estabelecido o papel da APS como campo de prática propício para MTC, e de já existirem vários estudos acerca de quais tipos dessas práticas estão sendo utilizadas, pontos abordados até mesmo pelos bancos de dados como o PMAQ e o Inquérito (BARBOSA, 2020), ainda existem poucos trabalhos que tem como foco quais os problemas de saúde estão sendo abordados nessa prática. Nessa perspectiva, busca-se neste estudo avaliar quais os agravos de saúde mais abordados pelas PICs no cenário brasileiro.

\section{Métodos}

O seguinte estudo trata-se de uma revisão integrativa de literatura na qual se buscou avaliar o perfil de emprego das PICs na abordagem dos diversos problemas de saúde na atenção primária. Esse método de estudo foi selecionado por permitir ao pesquisador a construção de um panorama da produção científica disponível acerca do tema e novas possibilidades de pesquisa (BOTELHO; CUNHA; MACEDO, 2011).

A estruturação baseou-se nos passos propostos por Botelho, Cunha e Macedo (2011), sendo composta por 6 etapas, que consistem em: escolha do tema e definição do problema de pesquisa; elaboração de critérios de inclusão e exclusão; identificação dos estudos selecionados; categorização desses estudos; análise e discussão dos resultados; apresentação e síntese da revisão.

Inicialmente, a partir da temática de PICs e APS foi observado como relevante analisar quais os principais problemas de saúde abordados pelo uso dessas práticas na APS. Com base nessa questão foram então selecionados os descritores "Terapias Complementares (Complementary therapies)" e “Atenção Primária à Saúde (Primary Health Care)", sendo então realizada pesquisa nas bases de dados PubMed e Biblioteca Virtual em Saúde (BVS). 
A pesquisa bibliográfica inicial teve como resultado a identificação de 371 artigos na Pubmed e 392 artigos na BVS, sendo então aplicados os seguintes critérios de elegibilidade: texto completo; idioma português; documentos publicados nos últimos 5 anos. Após aplicação dos filtros foram pré-selecionadas sete publicações na PubMed e 42 na BVS para leitura dos resumos, a partir dos quais, após exclusão de repetições e daqueles documentos que não respondiam à pergunta norteadora da pesquisa obteve-se a amostra final para avaliação na íntegra, que consistiu em 14 publicações no total. (Fluxograma 1).

Fluxograma 1: Processo de seleção das publicações sobre o objeto de estudo

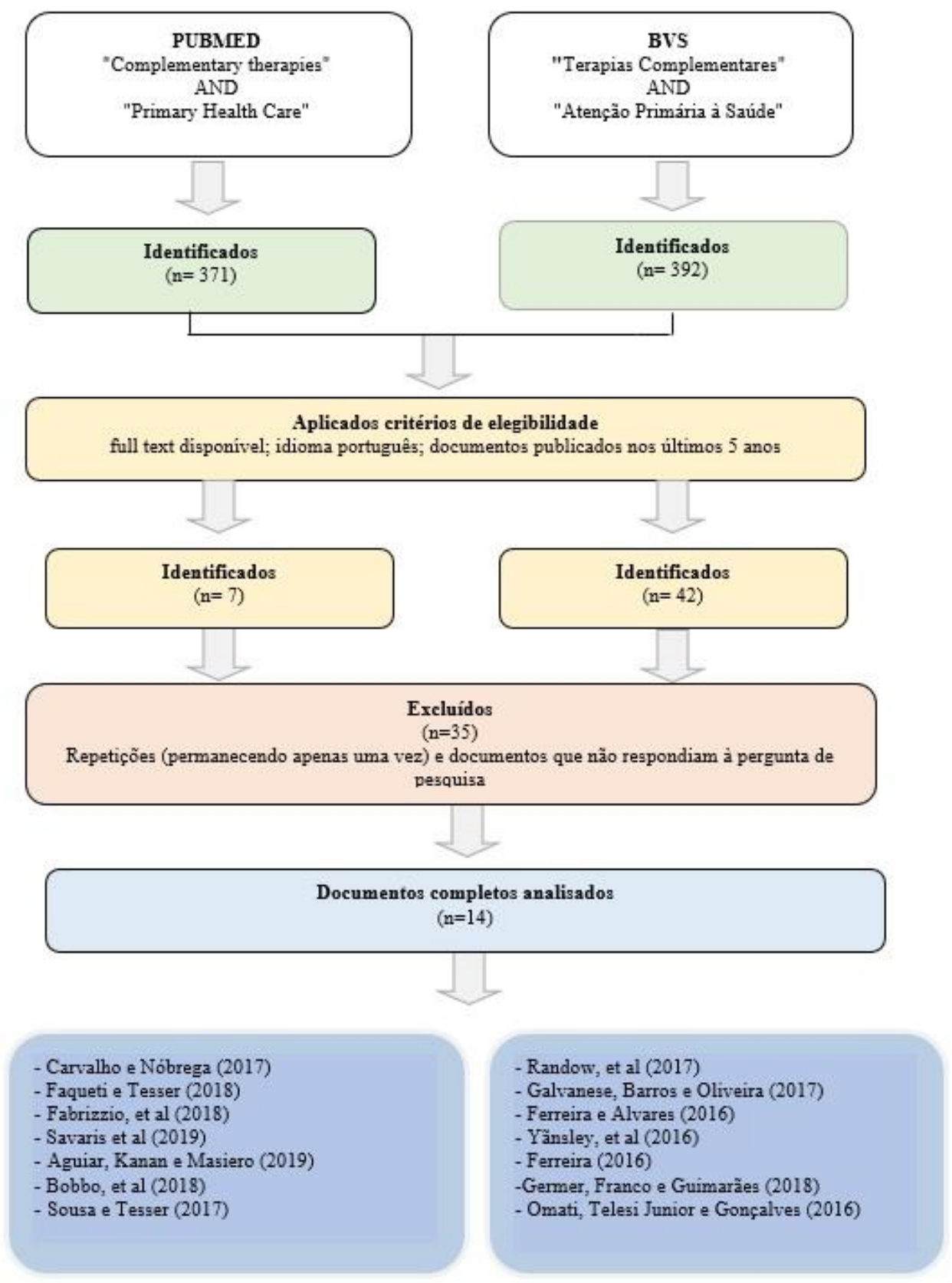

Fonte: dados da Pesquisa, 2020. 
Os artigos selecionados foram submetidos à leitura detalhada com avaliação e descrição da caracterização geral dos estudos, sendo avaliados seus principais achados e então categorizados de acordo com o grupo de problema de saúde abordado, sendo eles: saúde mental; manejo da dor; doenças crônicas; doenças neurológicas; menopausa.

\section{Resultados}

Os quadros a seguir demonstram os estudos selecionados, desde sua caracterização, incluindo metodologia de estudo empregada e população trabalhada, até os principais achados encontrados e suas categorizações.

Observa-se que apenas três estudos $(21,4 \%)$ tiveram uma abordagem quantitativa, com a maioria apresentando metodologia de caráter qualitativo e relatos de experiência, além de 1 estudo consistir em bibliometria. Verifica-se também que as populações abordadas nos estudos tiveram prevalência semelhante em relação ao número de estudos que abordaram profissionais de saúde e usuários do serviço de saúde, com 42,85\% cada. (Quadro 1).

Quadro 1: Caracterização dos estudos selecionados

\begin{tabular}{|c|c|c|c|c|c|c|}
\hline Autores/Ano & Título do Artigo & $\begin{array}{l}\text { Base de } \\
\text { Dados } \\
\end{array}$ & Revista & País & Método & $\begin{array}{l}\text { População do } \\
\text { estudo }\end{array}$ \\
\hline $\begin{array}{l}\text { Carvalho e Nóbrega } \\
\text { (2017) }\end{array}$ & $\begin{array}{l}\text { Terapias } \\
\text { complementares } \\
\text { como recursos para } \\
\text { a saúde mental na } \\
\text { Atenção Primária à } \\
\text { Saúde }\end{array}$ & $\begin{array}{l}\text { PUBMED } \\
\text { Scielo }\end{array}$ & $\begin{array}{l}\text { Revista } \\
\text { Gaúcha de } \\
\text { Enfermagem }\end{array}$ & Brasil & $\begin{array}{l}\text { Estudo } \\
\text { quantitativo }\end{array}$ & $\begin{array}{l}\text { Profissionais de } \\
\text { uma UBS na } \\
\text { cidade de São } \\
\text { Paulo }\end{array}$ \\
\hline $\begin{array}{l}\text { Faqueti eTesser } \\
\text { (2018) }\end{array}$ & $\begin{array}{l}\text { Utilização de } \\
\text { Medicinas } \\
\text { Alternativas e } \\
\text { Complementares na } \\
\text { atenção primária à } \\
\text { saúde de } \\
\text { Florianópolis/SC, } \\
\text { Brasil: percepção de } \\
\text { usuários }\end{array}$ & $\begin{array}{l}\text { PUBMED } \\
\text { Scielo }\end{array}$ & $\begin{array}{l}\text { Ciência \& } \\
\text { Saúde } \\
\text { Coletiva }\end{array}$ & Brasil & $\begin{array}{l}\text { Estudo } \\
\text { descritivo } \\
\text { qualitativo }\end{array}$ & $\begin{array}{l}\text { Usuários de PICs } \\
\text { atendidos por } \\
\text { equipes de APS } \\
\text { em } \\
\text { Florianópois/SC. }\end{array}$ \\
\hline $\begin{array}{l}\text { Fabrizzio et al. } \\
\text { (2018) }\end{array}$ & $\begin{array}{l}\text { Gestão do cuidado } \\
\text { de um paciente com } \\
\text { Doença de Devic na } \\
\text { Atenção Primária à } \\
\text { Saúde }\end{array}$ & $\begin{array}{l}\text { BVS } \\
\text { MEDLINE }\end{array}$ & $\begin{array}{l}\text { Revista da } \\
\text { Escola de } \\
\text { Enfermagem } \\
\text { da USP }\end{array}$ & Brasil & $\begin{array}{l}\text { Estudo de } \\
\text { caso } \\
\text { clínico- } \\
\text { qualitativo }\end{array}$ & $\begin{array}{l}\text { Paciente de um } \\
\text { centro de estudo } \\
\text { de serviço de } \\
\text { Florianópolis } \\
\text { acompanhado pela } \\
\text { APS }\end{array}$ \\
\hline
\end{tabular}




\begin{tabular}{|c|c|c|c|c|c|c|}
\hline $\begin{array}{l}\text { Savaris et al. } \\
(2019)\end{array}$ & $\begin{array}{l}\text { Práticas integrativas } \\
\text { e complementares - } \\
\text { análise documental e } \\
\text { o olhar de } \\
\text { profissionais da } \\
\text { saúde }\end{array}$ & $\begin{array}{l}\text { BVS } \\
\text { LILACS }\end{array}$ & $\begin{array}{l}\text { Revista } \\
\text { brasileira em } \\
\text { promoção da } \\
\text { saúde }\end{array}$ & Brasil & $\begin{array}{l}\text { Estudo } \\
\text { documental } \\
\text { retrospectivo } \\
\text { E } \\
\text { Quantitativo } \\
\text { transversal }\end{array}$ & $\begin{array}{l}\text { Profissionais de } \\
\text { UBSs de } \\
\text { Curitiba/PR }\end{array}$ \\
\hline $\begin{array}{l}\text { Aguiar, Kanan e } \\
\text { Masiero } \\
(2019)\end{array}$ & $\begin{array}{l}\text { Práticas Integrativas } \\
\text { e Complementares } \\
\text { na atenção básica } \\
\text { em saúde: um estudo } \\
\text { bibliométrico da } \\
\text { produção brasileira }\end{array}$ & $\begin{array}{l}\text { BVS } \\
\text { LILACS }\end{array}$ & $\begin{array}{l}\text { Saúde em } \\
\text { debate }\end{array}$ & Brasil & Bibliometria & $\begin{array}{l}\text { Produção científica } \\
\text { acerca de PICs } \\
\text { desenvolvida no } \\
\text { Brasil, entre } 2006 \text { e } \\
2016 .\end{array}$ \\
\hline $\begin{array}{l}\text { Bobbo et al. } \\
(2018)\end{array}$ & $\begin{array}{l}\text { Saúde, dor e } \\
\text { atividades de vida } \\
\text { diária entre idosos } \\
\text { praticantes de Lian } \\
\text { Gong e sedentários }\end{array}$ & $\begin{array}{l}\text { BVS } \\
\text { LILACS }\end{array}$ & $\begin{array}{l}\text { Ciência \& } \\
\text { saúde } \\
\text { coletiva }\end{array}$ & Brasil & $\begin{array}{l}\text { Estudo } \\
\text { transversal } \\
\text { quantitativo }\end{array}$ & $\begin{array}{l}\text { Praticantes } \\
\text { regulares de grupo } \\
\text { de Lian Gong em } \\
\text { uma unidade de } \\
\text { APS } \\
\end{array}$ \\
\hline $\begin{array}{l}\text { Sousa e Tesser } \\
(2017)\end{array}$ & $\begin{array}{l}\text { Medicina } \\
\text { Tradicional e } \\
\text { Complementar no } \\
\text { Brasil: inserção no } \\
\text { Sistema Único de } \\
\text { Saúde e integração } \\
\text { com a atenção } \\
\text { primária }\end{array}$ & $\begin{array}{l}\text { BVS } \\
\text { LILACS }\end{array}$ & $\begin{array}{l}\text { Cadernos de } \\
\text { saúde } \\
\text { pública }\end{array}$ & Brasil & $\begin{array}{l}\text { Estudo } \\
\text { qualitativo }\end{array}$ & $\begin{array}{l}\text { Estudos de caso e } \\
\text { pesquisas realizadas } \\
\text { em municípios } \\
\text { brasileiros com uso } \\
\text { de Medicina } \\
\text { Tradicional e } \\
\text { Complementar em } \\
\text { serviços públicos }\end{array}$ \\
\hline $\begin{array}{l}\text { Randow et al. } \\
(2017)\end{array}$ & $\begin{array}{l}\text { Lian Gong em } 18 \\
\text { terapias como } \\
\text { estratégia de } \\
\text { promoção da saúde }\end{array}$ & $\begin{array}{l}\text { BVS } \\
\text { LILACS }\end{array}$ & $\begin{array}{l}\text { Revista } \\
\text { brasileira em } \\
\text { promoção da } \\
\text { saúde }\end{array}$ & Brasil & $\begin{array}{l}\text { Estudo } \\
\text { transversal } \\
\text { descritivo }\end{array}$ & $\begin{array}{l}\text { Praticantes de lian } \\
\text { gong no município } \\
\text { de Belo } \\
\text { Horizonte/MG }\end{array}$ \\
\hline $\begin{array}{l}\text { Galvanese, Barros e } \\
\text { Oliveira } \\
(2017)\end{array}$ & $\begin{array}{l}\text { Contribuições e } \\
\text { desafios das práticas } \\
\text { corporais e } \\
\text { meditativas à } \\
\text { promoção da saúde } \\
\text { na rede pública de } \\
\text { atenção primária do } \\
\text { Município de São } \\
\text { Paulo, Brasil } \\
\end{array}$ & $\begin{array}{l}\text { BVS } \\
\text { LILACS }\end{array}$ & $\begin{array}{l}\text { Cadernos de } \\
\text { Saúde } \\
\text { Pública }\end{array}$ & Brasil & $\begin{array}{l}\text { Estudo } \\
\text { qualitativo }\end{array}$ & $\begin{array}{l}\text { Usuários de } \\
\text { unidades de saúde } \\
\text { do município de São } \\
\text { Paulo/SP }\end{array}$ \\
\hline $\begin{array}{l}\text { Ferreira e Alvares } \\
(2016)\end{array}$ & $\begin{array}{l}\text { Inserção da } \\
\text { acupuntura na } \\
\text { Atenção Básica } \\
\text { como tratamento } \\
\text { terapêutico } \\
\text { complementar das } \\
\text { doenças crônicas }\end{array}$ & BVS & $\begin{array}{l}\text { BIS: Boletim } \\
\text { do Instituto } \\
\text { de Saúde }\end{array}$ & Brasil & $\begin{array}{l}\text { Relato de } \\
\text { experiência }\end{array}$ & $\begin{array}{l}\text { UBSs do município } \\
\text { de Pompéia/SP }\end{array}$ \\
\hline $\begin{array}{l}\text { Yãnsley et al. } \\
(2016)\end{array}$ & $\begin{array}{l}\text { Qualidade de vida } \\
\text { de indivíduos } \\
\text { praticantes de } \\
\text { Qigong na } \\
\text { comunidade }\end{array}$ & BVS & $\begin{array}{l}\text { Revista } \\
\text { Extensão em } \\
\text { Ação }\end{array}$ & Brasil & $\begin{array}{l}\text { Estudo } \\
\text { transversal } \\
\text { descritivo }\end{array}$ & $\begin{array}{l}\text { Usuários do grupo } \\
\text { de Práticas Corpo- } \\
\text { Mente em UBS do } \\
\text { município de } \\
\text { Fortaleza/CE }\end{array}$ \\
\hline $\begin{array}{l}\text { Ferreira } \\
(2016)\end{array}$ & $\begin{array}{l}\text { Práticas integrativas } \\
\text { e complementares } \\
\text { (PICs) no cuidado } \\
\text { em saúde mental }\end{array}$ & BVS & $\begin{array}{l}\text { Repositório } \\
\text { Institucional } \\
\text { da UFSC }\end{array}$ & Brasil & $\begin{array}{l}\text { Tese } \\
\text { Estudo de } \\
\text { caso }\end{array}$ & $\begin{array}{l}\text { Profissionais de } \\
\text { saúde de duas UBSs } \\
\text { do município de } \\
\text { Florianópolis/SC }\end{array}$ \\
\hline
\end{tabular}




\begin{tabular}{l|l|l|l|l|l|l}
\hline $\begin{array}{l}\text { Germer, Franco e } \\
\text { Guimarães } \\
(2018)\end{array}$ & $\begin{array}{l}\text { As práticas } \\
\text { integrativas e } \\
\text { complementares } \\
\text { como elo integrador } \\
\text { dos cuidados em } \\
\text { saúde }\end{array}$ & BVS & $\begin{array}{l}\text { Sec. Munic. } \\
\text { Saúde SP }\end{array}$ & Brasil & $\begin{array}{l}\text { Relato de } \\
\text { experiência }\end{array}$ & $\begin{array}{l}\text { Equipe de NASF da } \\
\text { cidade de São } \\
\text { Paulo/SP }\end{array}$ \\
\hline $\begin{array}{l}\text { Omati, Telesi Junior e e } \\
\text { Gonçalves } \\
(2016)\end{array}$ & $\begin{array}{l}\text { Implantação da } \\
\text { craniopuntura de } \\
\text { Yamamoto na SMS- } \\
\text { SP }\end{array}$ & BVS & $\begin{array}{l}\text { Sec. Munic. } \\
\text { Saúde SP }\end{array}$ & Brasil & $\begin{array}{l}\text { Relato de } \\
\text { experiência }\end{array}$ & $\begin{array}{l}\text { Serviços de saúde } \\
\text { em São Paulo/SP }\end{array}$ \\
\hline
\end{tabular}

Fonte: dados da Pesquisa, 2020.

Os principais resultados levantados nos estudos evidenciam que o uso das PICS é aplicado principalmente em condições de agravo à saúde como diabetes mellitus (DM), hipertensão arterial sistêmica (HAS), distúrbios de humor e dor crônica. Além disso, $42 \%$ dos estudos apontam para o uso das PICs como fator que auxilia na diminuição da necessidade de uso de fármacos no tratamento de agravos à saúde. (Quadro 2)

Quadro 2: Principais achados dos estudos selecionados

\begin{tabular}{|c|c|}
\hline Autores/Ano & $\begin{array}{r}\text { Principais achados } \\
\end{array}$ \\
\hline $\begin{array}{l}\text { Carvalho e Nóbrega } \\
\text { (2017) }\end{array}$ & $\begin{array}{l}\text { - A maioria dos profissionais }(62,9 \%) \text { indicaria as PICS para o cuidado de questões } \\
\text { emocionais/mentais/comportamentais } \\
\text { - Os profissionais que tem conhecimento das práticas de Medicina Tradicional } \\
\text { Chinesa/Acupuntura/Massoterapia tendem a desacreditar que essas práticas possam } \\
\text { atrapalhar o tratamento convencional medicamentoso já instituído em Saúde Mental }\end{array}$ \\
\hline $\begin{array}{l}\text { Faqueti eTesser } \\
(2018)\end{array}$ & $\begin{array}{l}\text { - Os motivos que levaram à procura das PICs na população estudada foram } \\
\text { principalmente dores corporais e problemas relacionados à ansiedade, humor e } \\
\text { stress } \\
\text { - Metade da população entrevistada preferiu utilizar as PICs antes dos tratamentos } \\
\text { farmacológicos, indo além do seu uso alternativo ou complementar. }\end{array}$ \\
\hline $\begin{array}{l}\text { Fabrizzio et al. } \\
(2018)\end{array}$ & $\begin{array}{l}\text { - A doença de Devic é uma patologia rara, imunomediada, incluída no grupo de doenças } \\
\text { desmielinizantes inflamatórias idiopáticas. Pode apresentar recidivas de episódios. } \\
\text { - Os sintomas da paciente eram principalmente relacionados à dor e as crises ocorriam } \\
\text { em momentos de stress } \\
\text { - Após um ano de tratamento com acupuntura e auriculoterapia, associado ao } \\
\text { tratamento medicamentoso, foi possível estabilização do quadro. }\end{array}$ \\
\hline $\begin{array}{l}\text { Savaris et al. } \\
(2019)\end{array}$ & 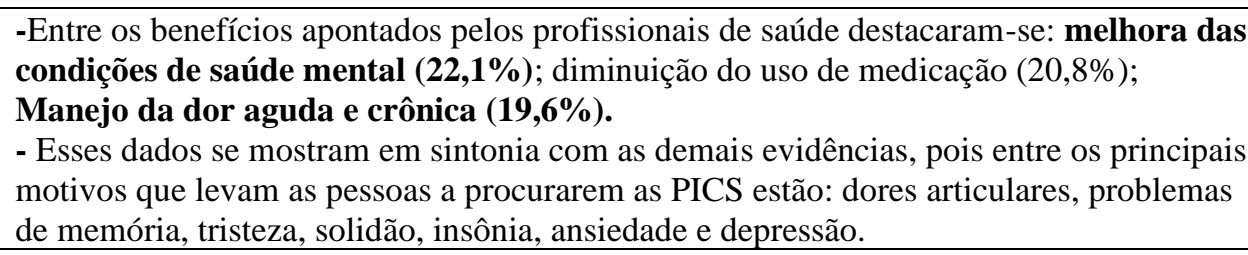 \\
\hline $\begin{array}{l}\text { Aguiar, Kanan e } \\
\text { Masiero } \\
(2019)\end{array}$ & $\begin{array}{l}\text { - O estudo destaca os resultados e principais características da utilização das PICS } \\
\text { disponíveis na bibliografia brasileira } \\
\text { - Entre os problemas de saúde tratados com PICS destacam-se: transtornos mentais } \\
\text { graves e leves; hipertensão, diabetes e outras doenças crônicas; insônia; sintomas } \\
\text { da menopausa e outros problemas de ordem ginecológica. }\end{array}$ \\
\hline
\end{tabular}


Bobbo et al.

(2018)
- O estudo foca na abordagem comparacional entre pacientes sedentários e pacientes praticantes de Lian Gong.

- Em relação à dor osteomuscular, embora não se tenha observado diferenças em relação ao nível total de dor nos dois grupos, para a região inferior das costas, a prática de Lian Gong esteve associada a uma menor sensação de restrição ao realizar atividades de vida diária

- O grupo de indivíduos ativos deste estudo apresentou menos problemas cardiovasculares e menor uso de medicamentos.

Sousa e Tesser

(2017)

- Quanto ao perfil de demanda em relação às PICS nas equipes de saúde da família pode-se destacar problemas de saúde mental, osteomusculares, dores em geral e doenças crônicas

- No presente estudo deu-se enfoque ao benefício do uso do Lian Gong no manejo da dor

Randow et al. (2017)

- Outros benefícios apontados foram a redução no uso de medicamentos e a redução da taxa de procura por outros serviços de saúde

- Os principais pontos apontados pelos usuários como melhora parcial ou total após a

Galvanese, Barros e Oliveira (2017) prática de PICS foram: dores articulares e mobilidade, além de memória (referidos principalmente por idosos); e sintomas de depressão e ansiedade, com melhor qualidade do sono.

- Além disso, pacientes que apresentavam diabetes e hipertensão arterial relataram maior equilíbrio nos parâmetros de controle destas condições e, em alguns casos, diminuição da necessidade de uso de fármacos.

Ferreira e Alvares

(2016)

- Relato de experiência acerca do uso de acupuntura no manejo da dor crônica em UBSs

- Além de melhora da dor percebeu-se também diminuição no uso de medicamentos e retorno à execução de atividades do dia-a-dia

- Entre as queixas relatadas pelos usuários do serviço destacaram-se queixas musculoesqueléticas (20\%), ansiedade (20\%) e insônia (13,3\%).

Yãnsley et al.

(2016)

- Se destaca a necessidade de se desenvolver ações na atenção básica que visem à integração da saúde física e mental

- Identificou-se benefícios do emprego das PICs principalmente em transtornos de ansiedade e do humor.

Ferreira

(2016)

- As PICs foram identificadas como importante recurso para reduzir a medicalização dos transtornos psíquicos pelos profissionais das unidades.

Germer, Franco e

Guimarães

(2018)

Omati, Telesi Junior e

Gonçalves

(2016)
- Foram relatados pelos usuários melhora das condições de sono, redução da dor, redução da ansiedade, melhora dos aspectos motores amplos e consciência corporal.

- Destaca-se os benefícios da prática para tratamento de dor que acomete todo o aparelho cinético (dorsalgia, lombalgia, gonalgia, fasceíte plantar, síndrome do túnel do carpo, dor no ombro, epicondilite)

- Tratamento de doenças neurológicas, principalmente para as sequelas causadas pelo acidente vascular cerebral (AVC), como a paralisia facial ou motora e a afasia, a paralisia cerebral em crianças

- Além disso, também há relatos de benefícios em insônia, ansiedade, depressão, labirintite, cefaleia tensional e enxaqueca

- Percebeu-se redução do uso de anti-inflamatórios em algumas Unidades de Saúde

Fonte: dados da Pesquisa, 2020.

O quadro 3 categoriza os problemas de saúde para os quais são utilizadas as PICS na APS, dividindo-os em cinco categorias. Observa-se que a grande maioria dos estudos considera a aplicação das terapias complementares no manejo da dor $(78,5 \%)$ e na saúde mental $(71,4 \%)$, com apenas $24 \%$ dos estudos considerando a aplicação dessas práticas no manejo de doenças 
crônicas orgânicas (como HAS e DM), 2 estudos abordando doenças neurodegenerativas e 1 estudo abordando a menopausa.

Quadro 3: Categorização do estudo

\begin{tabular}{|c|c|c|c|}
\hline Categoria & Autores/Ano & $\mathbf{N}$ & $\%$ \\
\hline Manejo da dor & $\begin{array}{l}\text { - Faqueti eTesser (2018) } \\
\text { - Fabrizzio et al. (2018) } \\
\text { - Savaris et al. (2019) } \\
\text { - Bobbo et al. (2018) } \\
\text { - Sousa e Tesser (2017) } \\
\text { - Randow et al. (2017) } \\
\text { - Galvanese, Barros e Oliveira (2017) } \\
\text { - Ferreira e Alvares (2016) } \\
\text { - Yãnsley et al. (2016) } \\
\text { - Germer, Franco e Guimarães (2018) } \\
\text { - Omati, Telesi Junior e Gonçalves (2016) }\end{array}$ & 11 & $\mathbf{7 8 , 5 7}$ \\
\hline Saúde Mental & $\begin{array}{l}\text { - Carvalho e Nóbrega (2017) } \\
\text { - Faqueti eTesser (2018) } \\
\text { - Savaris et al. (2019) } \\
\text { - Aguiar, Kanan e Masiero (2019) } \\
\text { - Sousa e Tesser (2017) } \\
\text { - Galvanese, Barros e Oliveira (2017) } \\
\text { - Yãnsley et al. (2016) } \\
\text { - Ferreira (2016) } \\
\text { - Germer, Franco e Guimarães (2018) } \\
\text { - Omati, Telesi Junior e Gonçalves (2016) }\end{array}$ & 10 & 71,42 \\
\hline Doenças crônicas & $\begin{array}{l}\text { - Aguiar, Kanan e Masiero (2019) } \\
\text { - Bobbo et al. (2018) } \\
\text { - Sousa e Tesser (2017) } \\
\text { - Galvanese, Barros e Oliveira (2017) }\end{array}$ & 4 & $\mathbf{2 8 , 5 7}$ \\
\hline Doenças Neurológicas & $\begin{array}{l}\text { - Fabrizzio et al. (2018) } \\
\text { - Omati, Telesi Junior e Gonçalves (2016) }\end{array}$ & 2 & 14,28 \\
\hline Menopausa & - Aguiar, Kanan e Masiero (2019) & 1 & 7,14 \\
\hline \multicolumn{2}{|r|}{ Total } & 14 & 100 \\
\hline
\end{tabular}

Fonte: dados da Pesquisa, 2020.

\section{Discussão}

Verifica-se prevalência de estudos qualitativos descritivos nessa área, abordando tanto a experiência de profissionais de saúde em relação ao tema como também os próprios usuários de saúde, além de ser possível enquadrar os problemas de saúde abordados em cinco categorias básicas, como descrito e referenciado no quadro 3: manejo da dor; saúde mental; doenças crônicas; doenças neurológicas e menopausa.

A prevalência da existência de estudos com metodologia qualitativa e descritiva pode ser atribuída tanto à própria complexidade inerente às PICS e a seu mecanismo de avaliação, visto que elas buscam não só a cura na análise de sua eficácia terapêutica (BARBOSA, 2020), 
como também pode demonstrar a falta de estímulo às pesquisas nessa área, sendo grande partes dos estudos compostos por relatos de experiência, o que se adequa à uma realidade na qual são os profissionais de saúde os pioneiros da implantação das PICS nos serviços, independente de financiamento da gestão pública (AGUIAR; KANAN; MASIERO, 2019).

O número semelhante de estudos que abordaram como população alvo tanto a perspectiva de profissionais de saúde como a dos pacientes entram em consenso com a valorização e legitimação social (TESSER; SOUSA; NASCIMENTO, 2018) que as PICs vêm adquirindo, sendo estas as populações que estão em contato direto na busca por estas práticas e seus resultados, que é estimulada por fatores como insatisfações com a limitação da medicina tradicional.

Em relação aos problemas de saúde abordados, a alta taxa de uso dessas práticas na abordagem do manejo da dor e saúde mental se relaciona ao próprio contexto cultural e histórico que fez as PICs e sua forma ampliada de escuta e cuidado ganharem destaque na atenção à saúde (SOUSA; HORTALE; BODSTEIN, 2018). No entanto, a baixa taxa de estudos que abordaram as doenças crônicas cardiovasculares e metabólicas como HAS e DM (apenas 24\%) vai de encontro à essa perspectiva e às características essenciais da MTC (GALVANESE; BARROS; OLIVEIRA, 2017). Vale ressaltar que o achado em questão não significa uma baixa eficácia das PICS no tratamento dessas patologias, pois como pode ser observado em estudo realizado por Pereira et al. (2017) que consistiu em revisão integrativa abordando a acupuntura no manejo da HAS, ocorreu a melhora da saúde através da autocura, podendo ser avaliada através da redução dos níveis pressóricos dos pacientes.

O próprio caráter multifatorial das doenças crônicas aponta para o possível benefício do uso das PICS no seu manejo, sendo que estas práticas estimulam o autocuidado e corresponsabilização do indivíduo no seu estado de saúde (GALVANESE; BARROS; OLIVEIRA, 2017). Além disso, a diminuição da necessidade do uso de fármacos, percebida em $42 \%$ dos estudos analisados, favorece a diminuição de iatrogenias e efeitos colaterais causados pelo tratamento farmacológico excessivo destas patologias.

Vale ressaltar, que apesar de ser a APS o principal campo das PICs no Brasil, a restrição da pesquisa e seleção dos artigos a esse campo de prática também excluiu da análise realizada os possíveis usos dessas práticas em outras patologias e agravos à saúde mais específicos, que geralmente são abordados na atenção secundária e terciária, o que limita de certa forma os resultados encontrados. 


\section{Conclusão}

Através do exposto, observa-se que as PICs, apesar de apresentarem características que são úteis principalmente ao manejo das doenças crônicas, nesse campo os estudos sobre sua aplicação ainda são escassos, estando a maioria dos estudos concentrada no campo da saúde mental e manejo da dor.

Além disso, outras patologias e condições mais específicas, também passíveis de abordagem através das PICS, como as doenças neurodegenerativas e a menopausa, apresentam estudos ainda mais escassos. Isso mostra a necessidade de estímulo e financiamento às pesquisas e avaliações sobre uso e resultados das PICS, tanto na APS como fora dela, principalmente a partir de experiências que já vem sendo desenvolvida e muitas vezes não são divulgadas, a fim de que seja possível o surgimento de uma base bibliográfica sólida que sustente o uso das PICS em diversas áreas do cuidado à saúde.

\section{Referências}

AGUIAR J.; KANAM L. A.; MASIERO A. V.. Práticas Integrativas e Complementares na atenção básica em saúde: um estudo bibliométrico da produção brasileira. Saúde debate [online], v. 43, n. 123, p. 1205-1218, out-dez 2019.

BARBOSA F. E. S., et al. Oferta de Práticas Integrativas e Complementares em Saúde na Estratégia Saúde da Família no Brasil. Cad. Saúde Pública, v. 36(1), e00208818, 2020.

BOBBO V. C. D., et al.. Saúde, dor e atividades de vida diária entre idosos praticantes de Lian Gong e sedentários. Ciência e saúde coletiva, v. 23, n. 4, p. 1151-1158, 2018.

BOTELHO L. L. R.; CUNHA C. C. A.; MACEDO M.. O método da revisão integrativa nos estudos organizacionais. Gestão e Sociedade, v. 5, n. 11, p. 121-136, 2014.

CARVALHO J. L. D. S.; NÓBREGA M. D. P. S. S.. Terapias complementares como recursos para a saúde mental na Atenção Primária à Saúde. Revista Gaúcha de Enfermagem, v. 38, n. 4, e2017-0014, 2017.

FABRIZZIO G. C.; et al.. Gestão do cuidado de um paciente com Doença de Devic na Atenção Primária à Saúde. Revista da Escola de Enfermagem da USP, v. 52, e03345, 2018.

FAQUETI A.; TESSER C. D.. Utilização de Medicinas Alternativas e Complementares na atenção primária à saúde de Florianópolis/SC, Brasil: percepção de usuários. Ciência \& Saúde Coletiva, v. 23, n. 8, p. 2621-2630, 2018.

FERREIRA D. D.. Práticas integrativas e complementares (PICs) no cuidado em saúde mental. Repositório Institucional da UFSC, 2016.

FERREIRA N. C.; ALVARES A. L. T.. Inserção da acupuntura na Atenção Básica como tratamento 
terapêutico complementar das doenças crônicas. BIS: Boletim do Instituto de Saúde, v. 17, p. 11-13. 2016.

GALVANESE A. T. C.; BARROS N. F.; OLIVEIRA A.. F. P. L.. Contribuições e desafios das práticas corporais e meditativas à promoção da saúde na rede pública de atenção primária do Município de São Paulo, Brasil. Cad. Saúde Pública [online], v. 33, n. 12, e00122016, 2017.

GERMER A. L. B.; FRANCO L.; GUIMARÃES D. X. D.. As práticas integrativas e complementares como elo integrador dos cuidados em saúde. Sec. Munic. Saúde SP, 2018.

Ministério da Saúde (BR). PORTARIA N ${ }^{\circ}$ 702, DE 21 DE MARÇO DE 2018. Altera a Portaria de Consolidação ${ }^{\circ}$ 2/GM/MS, de 28 de setembro de 2017, para incluir novas práticas na Política Nacional de Práticas Integrativas e Complementares - PNPIC. Diário Oficial da União, edição: 56, seção 1:65, mar 2018.

Ministério da Saúde (BR). Secretaria de Atenção à Saúde. Departamento de Atenção Básica. Política nacional de práticas integrativas e complementares no SUS: atitude de ampliação de acesso. Departamento de Atenção Básica, 2. ed., 2015.

OMATI R. S.; JUNIOR E. T.; GOLÇAVES R. C.. Implantação da craniopuntura de Yamamoto na SMSSP. Sec. Munic. Saúde SP - XXX Congresso de SecretáriosMunicipais de Saúde do Estado de São Paulo, 2016.

PEREIRA R. D.; et al. Acupuntura na hipertensão arterial sistêmica e suas contribuições sobre diagnósticos de enfermagem. Esc. Anna Nery [online], v. 21, n.1, e20170024, 2017.

RANDOW R., et al. Lian Gong em 18 terapias como estratégia de promoção da saúde. Revista brasileira em promoção da saúde, $\mathrm{v}$ 30, 2017.

SAVARIS L. E.; et al.. Práticas integrativas e complementares - análise documental e o olhar de profissionais da saúde. Revista brasileira em promoção da saúde, v. 32, 2019.

SOUSA I. M. C.; HORTALE V. A.; BODSTEIN R. C. A.. Medicina Tradicional Complementar e Integrativa: desafios para construir um modelo de avaliação do cuidado. Ciênc. saúde coletiva, V. 23, n.10, p. 3403-3412, 2018.

SOUSA I. M. C.; TESSER C. D.. Medicina Tradicional e Complementar no Brasil: inserção no Sistema Único de Saúde e integração com a atenção primária. Cadernos de saúde pública, v. 33, n.1, e00150215, 2017.

TESSER C. D.; SOUSA I. M. C.; NASCIMENTO M. C.. Práticas Integrativas e Complementares na Atenção Primária à Saúde brasileira. Saúde debate [online], v. 42, n. 1, p 174-188. 2018.

TESSER C D. Práticas complementares, racionalidades médicas e promoção da saúde: contribuições pouco exploradas. Cad. Saúde Pública, v 25, n. 8, p. 1732-1742, 2009.

YÃNSLEY A. T.; et al.. Qualidade de vida de indivíduos praticantes de Qigong na comunidade. Revista Extensão em Ação, v 1, n. 13, 2016.

\section{Como citar este artigo (Formato ABNT):}

VIDAL, Mariana dos Santos Araujo; SOUSA, Milena Nunes Alves de; TOLEDO, Miguel Aguila. Aplicação das Práticas Integrativas e Complementares na Atenção Primária à Saúde. Id on Line Rev.Mult. Psic., Fevereiro/2021, vol.14, n.54, p. 357-368. ISSN: 1981-1179.

Recebido: 21/01/2021; Aceito: 05/02/2021 